Bangladesh J. Plant Taxon. 16(2): 115-140, 2009 (December)

(C) 2009 Bangladesh Association of Plant Taxonomists

\title{
AN ASSESSMENT OF THE ANGIOSPERMIC FLORA OF RAMGARH UPAZILA OF KHAGRACHHARI DISTRICT, BANGLADESH
}

\author{
MD. RAFIQUL ISLAM ${ }^{1}$, MOHAMMAD ZASHIM UdDIN ${ }^{2}$ AND MD. ABUL HASSAN \\ Department of Botany, University of Dhaka, Dhaka 1000, Bangladesh.
}

Keywords: Assessment; Angiospermic flora; Khagrachhari; Bangladesh.

\begin{abstract}
The paper focuses on the qualitative assessment of angiospermic flora of Ramgarh upazila of Khagrachhari district conducted during May 2005 to September 2006. A total of 243 species belonging to 195 genera under 75 families were recorded. Magnoliopsida is represented by 60 families, 156 genera and 192 species, whereas Liliopsida by 15 families, 39 genera and 51 species. Fabaceae is the largest family in Magnoliopsida represented by 16 species and, in Liliopsida, Poaceae is the largest family with 17 species.
\end{abstract}

\section{Introduction}

Ramgarh upazila of Khagrachhari district is a part of greater Chittagong Hill Tracts of Bangladesh. The upazila lies between $22^{\circ} 51^{\prime}$ and $23^{\circ} 02^{\prime} \mathrm{N}$ latitudes and $91^{\circ} 43^{\prime}$ and $91^{\circ} 59^{\prime}$ E longitudes. It is bounded by Indian state of Tripura and Matiranga upazila of Bangladesh on the north, Manikchhari and Lakshmichhari upazilas on the south, Mahalchhari upazila on the east and Fatikchhari upazila on the west. Total area of the upazila is about 207.69 sq km (Lavlu, 2003).

Ramgarh upazila consists of many hills of different altitudes with an average elevation of $180 \mathrm{~m}$ above the sea level (Rizvi, 1969). The soil is reddish-yellowish sandy or sandy loam, mixed with scattered magniferous iron ore, huge humus throughout the forest but its degree of accumulation varies from place to place depending on topography, usually more deposition is found on flat land and on the bed of chhari (hilly streams) and less on the undulating hills. At high altitude, soil is compact and hard when it is dry; but soil is sandy and soft at low altitude. Soil pH varies from 4.5 to 6.0 (Rizvi, 1969). The climate is sub-tropical, with a long dry season extending from November to May, punctuated by largely unpredictable periods of rainstorm from June to September, the south-west monsoon provide the majority of the average annual rainfall of about 1815 $\mathrm{mm}$. Maximum temperature is $38.8^{\circ} \mathrm{C}$ recorded in April and minimum temperature is $9^{\circ} \mathrm{C}$ recorded in January (Bangladesh Meteorological Department, personal communication). The humidity on the whole is very high throughout the year.

Once the vegetation of Ramgarh upazila belonged to an evergreen and semievergreen forest (Choudhury, 1975). With the changing of time such vegetations are cleared. Now the flora of the upazila represented by homestead gardens, road-side plantations, monocultural plantation in the denuded hills, remaining scraft bushy jungles, bamboo bushes, sun grasses, tea estates, rubber plantations, and fruit gardens.

\footnotetext{
${ }^{1}$ E-mail: suhavedu@yahoo.com

${ }^{2}$ Corresponding author. E-mail: zashim07@yahoo.com
} 
Although there have been some published works (Heinig, 1925; Khan and Banu, 1969, 1972; Uddin et al., 1998) on the flora of the Chittagong Hill Tracts, no work exclusively on the Ramgarh upazila is available. To assess the angiospermic flora of Ramgarh upazila before further loss of present biodiversity of the area, the present attempt has been made.

\section{Materials and Methods}

The work is based on fresh materials collected during four visits to Ramgarh upazila from May 2005 to September 2006 to cover the seasonal variations. The visits covered all types of habitats, particularly hill-top, slope, foot-hills, valleys, village grove, fruit gardens and streams of the study area. Each trip lasted for eight days. Plant parts with either flowers or fruits collected using traditional herbarium techniques to make voucher specimens for documentation. Field identification of the collected specimens was confirmed comparing with herbarium specimens at Dhaka University Herbarium (DUH) and Bangladesh National Herbarium (DACB). In some cases, standard literature such as Hooker (1872-1897), Prain (1903), and Uddin and Hassan (2004) were consulted for identification purpose. The specimens are deposited in the Dhaka University Herbarium (DUH) for future reference.

\section{Results and Discussion}

In the present survey, a total of 243 angiospermic species under 195 genera and 75 families were recorded for Ramgarh upazila. Magnoliopsida is represented by 60 families, 156 genera and 192 species, while Liliopsida is represented by 15 families, 39 genera and 51 species. Habit-wise itemization of plant species shows that $36 \%$ of the total species are represented by herbs, $30 \%$ by trees, $22 \%$ by shrubs, $10 \%$ by climbers, and $2 \%$ by epiphytes and parasites.

The families have been arranged according to Cronquist (1981). The genera under each family and the species under each genus are arranged in an alphabetic order. For each species, nomenclature has been brought up-to-date; local name(s) (wherever available) and a short annotation are also provided. Tippara, Marma, and Chakma (major indigenous communities of the area) and Bangla names of the species are indicated by (t), (m), (c) and (b), respectively.

\section{Magnoliaceae}

\section{Magnoliopsida (Dicots)}

Michelia champaca L., Sp. Pl.: 536 (1753). Local name: Changab (m). A large or middle-sized tree. Representative specimen: Halfchhari, 07.05.05, Rafiqul Islam 29 (DUH). 


\section{Annonaceae}

Annona squamosa L., Sp. Pl.: 537 (1753). Local name: Sharifa (b). A small tree. Representative specimen: Halfchhari, 25.12.05, Rafiqul Islam 203 (DUH). Cultivated.

Uvaria hamiltonii Hook. f. \& Thoms., Fl. Ind. 1: 96 (1820). Local names: Kola (b), Tufaru (t). A large climber. Representative specimens: Halfchhari, 07.05.05, Rafiqul Islam 4 (DUH); Ramgarh, 11.05.05, Rafiqul Islam 152 (DUH).

\section{Lauraceae}

Cinnamomum camphora Prain, Beng. Pl. 2: 673 (1903). Local name: Karpur (b). A medium tree. Representative specimen: Pathachhara, 09.05.05, Rafiqul Islam 83 (DUH). Cultivated.

Dehaasia kurzii King ex Hook. f., Fl. Brit. Ind. 5: 125 (1886). Local name: Modon mosto (b). A tall tree. Representative specimen: Ramgarh, 11.05.05, Rafiqul Islam 135 (DUH).

Litsea monopetala (Roxb.) Pers., Syn. Pl. 2 (1): 4 (1807). Tetranthera monopetala Roxb., Pl. Corom. 2: 26 (1800). Local name: Menda buphang (t). A medium-sized tree. Representative specimen: Ramgarh, 09.09.06, Rafiqul Islam 270 (DUH).

\section{Piperaceae}

Piper longum L., Sp. Pl. 1: 28 (1753). Local name: Pepul (b). A perennial herb, branches with swollen nodes. Representative specimen: Halfchhari, 25.12.05, Rafiqul Islam 212 (DUH).

Piper nigrum L., Sp. Pl. 1: 28 (1753). Local name: Gol marich (b). A climber. Representative specimens: Pathachhara, 10.05.05, Rafiqul Islam 118 (DUH); Ramgarh, 21.12.05, Rafiqul Islam 168 (DUH). Cultivated.

\section{Aristolochiaceae}

Aristolochia tagala Cham., Linnaea 7: 207 (1832). Local name: Ishwarmul (b). A glabrous climber. Representative specimens: Pathachhara, 11.09.06, Rafiqul Islam 344 (DUH); Ramgarh, 11.05.05, Rafiqul Islam 133 (DUH).

\section{Menispermaceae}

Stephania japonica (Thunb.) Miers, Ann. Mag. Nat. Hist. Ser. 3, 18: 14 (1866). Menispermum japonicum Thunb., Fl. Jap.: 193 (1784). Local name: Maknadi (b). A slender climber. Representative specimen: Halfchhari, 17.09.06, Rafiqul Islam 383 (DUH). 
Tinospora sinensis (Lour.) Merr., Sunyatsenia 1: 193 (1934). Campylus sinensis Lour., Fl. Cochinch.: 113 (1790). Local name: Padmo gulancha (b). A climbing shrub. Representative specimen: Halfchhari, 25.12.05, Rafiqul Islam 219 (DUH).

\section{Papaveraceae}

Argemone mexicana L., Sp. Pl. 508 (1753). Local name: Sialkanta (b). An annual herb. Representative specimen: Ramgarh, 11.05.05, Rafiqul Islam 155 (DUH).

\section{Ulmaceae}

Trema orientalis (L.) Bl., Mus. Bot. Lugd.-Bat. 2: 63 (1856). Celtis orientalis L., Sp. Pl.: 1044 (1753). Local names: Bonanalia (b), Narsa (b). An ever-green, small tree. Representative specimens: Halfchhari, 25.12.05, Rafiqul Islam 201 (DUH); Pathachhara, 09.05.05, Rafiqul Islam 87 (DUH).

\section{Moraceae}

Artocarpus chaplasha Roxb., Fl. Ind. 3: 525 (1832). Local names: Champlate (t), Chapalish (b). A lofty, deciduous tree. Representative specimen: Pathachhara, 09.05.05, Rafiqul Islam 90 (DUH).

Artocarpus heterophyllus Lamk., Encycl. Meth. 3: 209 (1789). Local name: Kanthal (b). An ever-green tree. Representative specimen: Ramgarh, 09.05.05, Rafiqul Islam 39 (DUH). Cultivated.

Ficus heterophylla L. f., Suppl. Pl.: 442 (1781). Local name: Bhuidumur (b). A hispid, scandent shrub. Representative specimen: Halfchhari, 07.05.05, Rafiqul Islam 09 (DUH).

Ficus hirta Vahl, Enum. Pl. 2: 201 (1806). Local name: Pakur (b). A bushy tree. Representative specimen: Ramgarh, 09.05.05, Rafiqul Islam 68 (DUH).

Ficus hispida L. f., Suppl. Pl.: 442 (1781). Local name: Kakdumur (b). A low tree. Representative specimen: Ramgarh, 09.05.05, Rafiqul Islam 42 (DUH).

Ficus infectoria Roxb., Fl. Ind. 2: 643 (1824). Local names: Pakur (b), Takthu (t). A giant, spreading tree. Representative specimen: Ramgarh, 09.05.05, Rafiqul Islam 38 (DUH).

Ficus nervosa Heyne ex Roth in Roem. et Schult., Syst. Veg. 1: 513 (1817). A large tree. Representative specimen: Halfchhari, 09.05.05, Rafiqul Islam 71 (DUH).

Ficus religiosa L., Sp. Pl.: 1059 (1753). Local names: Ashathwa, Peepal (b). A large tree. Representative specimens: Pathachhari, 09.05.05, Rafiqul Islam 88 (DUH); Ramgarh, 17.09.06, Rafiqul Islam 354 (DUH). 


\section{Urticaceae}

Dendrocnide sinuata (Blume) Chew, Gard. Bull. Singh. 21: 206 (1965). Local name: Chutrapata (b). A shrub. Representative specimen: Ramgarh, 09.09.06, Rafiqul Islam 291 (DUH).

Sarcochlamys pulcherrima Gaud., Voy. Bot. t. 89 (1826). A shrub or small tree. Representative specimens: Halfchhari, 07.05.05, Rafiqul Islam 02 (DUH); Pathachhari, 11.09.06, Rafiqul Islam 351 (DUH).

Streblus asper Lour., Fl. Cochin. 2: 615 (1790). Local name: Shaora (b). A bushy tree. Representative specimen: Pathachhara, 22.12.05, Rafiqul Islam 193 (DUH).

\section{Fagaceae}

Quercus semiserrata Roxb., Fl. Ind. ed. 2, 3: 641 (1832). Local names: Goorja-batna, Rai-batna (b). A medium to large-sized tree. Representative specimen: Pathachhara, 22.12.05, Rafiqul Islam 188 (DUH).

\section{Amaranthaceae}

Alternanthera sessilis (L.) DC., Cat. Pl. Hort. Monspel.: 77 (1813). A herb, usually decumbent or prostrate. Representative specimen: Ramgarh, 09.09.06, Rafiqul Islam 224 (DUH).

Amaranthus spinosus L., Sp. Pl. 1: 991 (1753). Local name: Kanta-note (b). An annual, erect, spinescent herb. Representative specimen: Ramgarh, 21.12.05, Rafiqul Islam 176 (DUH).

Amaranthus viridis L., Sp. Pl. ed. 2: 1405 (1753). Local name: Note sak (b). An annual, erect or decumbent, small, slender herb. Representative specimen: Ramgarh, 09.05.05, Rafiqul Islam 49 (DUH).

\section{Polygonaceae}

Persicaria hydropiper (L.) Spach., Hist. Veg. 10: 536 (1841). Polygonum hydropiper L., Sp. Pl.: 361 (1753). Local name: Pakurmul (b). An annual herb. Representative specimens: Halfchhari, 17.09.06, Rafiqul Islam 385 (DUH); Ramgarh, 11.05.05, Rafiqul Islam 139 (DUH).

Persicaria minor (Huds.) Opiz, Seenam, Rosplin, Kbeteny, Ceske: 72 (1852). Polygonum minus Huds., Fl. Angl. 1: 148 (1762). An annual, erect or ascending herb. Representative specimen: Pathachhara, 09.05.05, Rafiqul Islam 84 (DUH).

\section{Dilleniaceae}

Dillenia indica L., Sp. Pl.: 535 (1753). Local name: Chalta (b). A tree. Representative specimen: Ramgarh, 09.05.05, Rafiqul Islam 69 (DUH). 


\section{Dipterocarpaceae}

Dipterocarpus alatus Roxb., Fl. Ind. 2: 614 (1824). Local name: Dhulia garjan (b). A tall tree. Representative specimen: Ramgarh, 21.12.05, Rafiqul Islam 166 (DUH).

Dipterocarpus turbinatus Gaertn., De Fruct. 3: 51 (1805). Local name: Garjan (b). A lofty tree. Representative specimen: Ramgarh, 11.05.05, Rafiqul Islam 136 (DUH).

\section{Theaceae}

Schima wallichii Choisy, Mem. Soc. Phys. Genev. 14: 144 (1855). Local name: Bonak (t). A large tree. Representative specimens: Halfchhari, 17.09.06, Rafiqul Islam 6 (DUH); Ramgarh, 17.09.06, Rafiqul Islam 369 (DUH).

\section{Clusiaceae (Guttiferae)}

Garcinia cowa Roxb., Fl. Ind. 2: 622 (1824). Local name: Kowphal (b). A tree. Representative specimens: Halfchhari, 17.09.06, Rafiqul Islam 390 (DUH); Pathachhara, 09.05.05, Rafiqul Islam 77 (DUH).

\section{Elaeocarpaceae}

Elaeocarpus tectorius Poir., Enc. Suppl. 2: 704 (1812). Local name: Jalpai (b). A tree. Representative specimen: Ramgarh, 21.12.05, Rafiqul Islam 167 (DUH). Cultivated.

\section{Tiliaceae}

Microcos paniculata L., Sp. Pl. 1: 514 (1753). Local names: Assar, Dattoi (b). A shrub to small tree. Representative specimen: Halfchhari, 07.05.05, Rafiqul Islam 36 (DUH).

\section{Sterculiaceae}

Abroma augusta (L.) L. f., Suppl. Pl.: 341 (1781). Theobroma augusta L., Syst. ed. 12: 233 (1767). Local name: Ulatkambal (b). A shrub. Representative specimens: Halfchhari, 25.12.05, Rafiqul Islam 220 (DUH); Pathachhara, 09.09.05, Rafiqul Islam 89 (DUH). Cultivated.

Buettneria pilosa Roxb., Fl. Ind. 2: 681 (1824). Local name: Harbhanga lata (b). A climbing shrub. Representative specimen: Pathachhara, 22.12.05, Rafiqul Islam 192 (DUH).

Firmiana colorata (Roxb.) R. Br. in Benn., Pl. Jav. Rar.: 235 (1844). Sterculia colorata Roxb., Pl. Corom. 1: 26, t. 25 (1795). Local name: Udal (b). A medium-sized tree. Representative specimen: Ramgarh, 09.05.05, Rafiqul Islam 50 (DUH). 


\section{Bombacaceae}

Bombax ceiba L., Sp. Pl.: 511 (1753). Local name: Shimul tula (b). A large tree with buttress base. Representative specimens: Halfchhari, 17.09.06, Rafiqul Islam 376 (DUH); Ramgarh, 21.12.05, Rafiqul Islam 180 (DUH).

\section{Malvaceae}

Hibiscus sabdariffa L., Sp. Pl.: 695 (1753). An annual herb. Representative specimen: Halfchhari, 07.05.05, Rafiqul Islam 8 (DUH). Cultivated.

Sida cordata (Burm. f.) Boriss., Blumea 14 (1): 182 (1966). Melochia cordata Burm. f., Fl. Ind. 143 (1768). Local name: Junka (b). An annual, slender, prostrate or ascending herb. Representative specimen: Pathachhara, 11.09.06, Rafiqul Islam 347 (DUH).

Urena lobata L., Sp. Pl.: 692 (1753). Local names: Banokra (t), Pungi (m). An undershrub. Representative specimen: Halfchhari, 07.05.05, Rafiqul Islam 7 (DUH).

\section{Lecythidaceae}

Careya arborea Roxb., Pl. Corom. 3: 14, t. 218 (1811). Local names: Gadila (m), Kamba (t). A low tree. Representative specimen: Pathachhara, 09.05.05, Rafiqul Islam 86 (DUH).

\section{Flacourtiaceae}

Flacourtia jangomas (Lour.) Raeusch., Nom. Bot. ed. 3: 290 (1797). Stigmarota jangomas Lour., Fl. Cochinch.: 634 (1790). Local name: Pina gola (b). A small, evergreen tree. Representative specimens: Halfchhari, 25.12.05, Rafiqul Islam 258 (DUH); Pathachhara, 09.05.05, Rafiqul Islam 79 (DUH).

\section{Bixaceae (Cochlospermataceae)}

Bixa orellana L., Sp. Pl.: 512 (1753). Local names: Cowabupang (t), Pahari lotka (b). A small, ever-green tree. Representative specimen: Halfchhari, 17.09.06, Rafiqul Islam 370 (DUH). Cultivated.

\section{Passifloraceae}

Passiflora foetida L., Sp. Pl.: 959 (1753). Local names: Jhumkolata (b), Pokki (t). A climbing, slender shrub. Representative specimen: Ramgarh, 11.05.05, Rafiqul Islam 138 (DUH). 


\section{Cucurbitaceae}

Coccinia grandis (L.) Voigt, Hort. Suburb. Calcut.: 59 (1845). Bryonia grandis L., Mant. Pl. 1: 126 (1767). Local names: Kawajhinga (t), Telakucha (b). A climber. Representative specimen: Ramgarh, 11.09.06, Rafiqul Islam 130 (DUH).

\section{Sapotaceae}

Achras sapota L., Sp. Pl. ed. 2: 470 (1753). Local name: Safeda (b). A medium-sized tree. Representative specimen: Pathachhara, 11.09.06, Rafiqul Islam 265 (DUH). Cultivated.

Madhuca longifolia (Koenig) Macbride, Contrib. Gray Herb. n. s. 53: 17 (1918). Bassia longifolia Koenig (1771). A long tree. Representative specimen: Ramgarh, 09.05.05, Rafiqul Islam 32 (DUH).

\section{Myrsinaceae}

Ardisia solanacea Roxb., Pl. Corom.: 27, t. 27 (1795). Local name: Banjam (b). An erect shrub. Representative specimen: Halfchhari, 25.12.05, Rafiqul Islam 221 (DUH).

\section{Mimosaceae}

Acacia concinna (Willd.) DC., Prodr. 2: 464 (1825). Mimosa concinna Willd., Sp. Pl. 4: 1039 (1805). Local name: Banritha (b). A bushy, spiny climber. Representative specimen: Ramgarh, 11.05.05, Rafiqul Islam 162 (DUH).

Acacia nilotica (L.) Delile subsp. indica (Benth.) Brenan in Kew Bull. 12: 84 (1957). Mimosa nilotica L., Sp. Pl.: 152 (1753). Local name: Babul (b). A tree. Representative specimens: Halfchhari, 25.12.05, Rafiqul Islam 211 (DUH); Pathachhara, 09.05.05, Rafiqul Islam 75 (DUH). Cultivated.

Adenanthera pavonina L., Sp. Pl. 1: 377 (1953). Local name: Raktachandan (b). A medium to large, deciduous tree. Representative specimen: Pathachhara, 22.12.05, Rafiqul Islam 194 (DUH). Cultivated.

Albizia chinensis (Osb.) Merr., Amer. J. Bot. 3: 575 (1916). Mimosa chinensis Osb., Degbok Ostind. Resa.: 233 (1757). Local names: Koroi (b), Kulmabuphang, Mashkala (t). A tall tree. Representative specimen: Pathachhara, 11.09.06, Rafiqul Islam 315 (DUH).

Albizia procera Benth. in Hook., London J. Bot. 3: 89 (1844). Local name: Buth koroi (b). A medium-sized tree. Representative specimens: Pathachhara, 11.09.06, Rafiqul Islam 304 (DUH); Ramgarh, 09.05.05, Rafiqul Islam 59 (DUH).

Mimosa pudica L., Sp. Pl.: 518 (1753). Local name: Lojjabati (b). A prickly, woody herb. Representative specimen: Halfchhari, 25.12.05, Rafiqul Islam 202 (DUH). 
Pithecellobium angulatum Benth. in Hook., London J. Bot. 3: 208 (1844). A short tree. Representative specimen: Pathachhara, 11.09.06, Rafiqul Islam 336 (DUH).

\section{Caesalpiniaceae}

Bauhinia scandens L., Sp. Pl.: 344 (1753). Local names: Gundilata (b), Kanson (t). An extensive, ever-green, woody climber. Representative specimen: Ramgarh, 11.05.05, Rafiqul Islam 140 (DUH).

Caesalpinia pulcherrima (L.) Swartz., Obs. Bot. Ind. Occ.: 166 (1791). Poinciana pulcherrima L. (1751). Local name: Radhachura (b). An unarmed, handsome shrub. Representative specimen: Halfchhari, 07.05.05, Rafiqul Islam 30 (DUH). Cultivated.

Cassia fistula L., Sp. Pl.: 377 (1753). Local names: Askhi, Badarlathi (b). A short tree. Representative specimen: Ramgarh, 09.05.05, Rafiqul Islam 57 (DUH).

Delonix regia Rafin., Fl. Tellur. 2: 92 (1836). Local names: Golmohar, Krishnachura (b). A medium-sized, handsome, deciduous tree. Representative specimen: Halfchhari, 07.05.05, Rafiqul Islam 25 (DUH). Cultivated.

Senna sophera (L.) Roxb., Fl. Ind. 2: 347 (1832). Cassia sophera L., Sp. Pl.: 379 (1753). Local names: Chotokalkesunde (b), Jhingi (t). A much branched shrub or undershrub. Representative specimen: Halfchhari, 25.12.05, Rafiqul Islam 204 (DUH).

Senna tora (L.) Roxb., Fl. Ind. 2: 340 (1832). Cassia tora L., Sp. Pl: 376 (1753). Local name: Lasiabupang (t). A perennial, erect, foetid, often profusely branched herb or undershrub. Representative specimen: Ramgarh, 09.09.06, Rafiqul Islam 275 (DUH).

Tamarindus indica L., Sp. Pl.: 34 (1753). Local name: Tentul (b). A tree. Representative specimen: Pathachhara, 10.05.05, Rafiqul Islam 114 (DUH).

\section{Fabaceae (Papilionaceae)}

Aeschynomene indica L., Sp. Pl.: 713 (1753). Local name: Katshola (b). Erect herb or undershrub. Representative specimens: Halfchhari, 17.09.06, Rafiqul Islam 365 (DUH); Ramgarh, 11.05.05, Rafiqul Islam 160 (DUH).

Atylosia scarabaeoides Benth. in Miq., Pl. Jungh. 1: 242 (1852). Local name: Banurkala (b). A herbaceous twiner. Representative specimen: Halfchhari, 25.12.05, Rafiqul Islam 199 (DUH).

Cajanus cajan (L.) Millsp., Columb. Mus. Bot. 2: 53 (1900). Cytisus cajan L., Sp. Pl.: 739 (1753). Local name: Orhor (b). A shrub. Representative specimen: Ramgarh, 11.05.05, Rafiqul Islam 126 (DUH). Cultivated.

Crotalaria pallida Aiton, Hort. Kew. 2: 20 (1789). Local names: Bara jhanjhani, Dangkok (t). An annual herb. Representative specimen: Pathachhara, 11.09.06, Rafiqul Islam 326 (DUH). 
Dalbergia elegans Benth. in Miq., Pl. Jungh. 1: 252 (1852). Local name: Singribish lata (b). A climber. Representative specimen: Halfchhari, 17.09.06, Rafiqul Islam 360 (DUH). Cultivated.

Dalbergia sissoo Roxb., Fl. Ind. 3: 223 (1832). Local names: Shimaki (t), Sisso (b). A tree. Representative specimen: Ramgarh, 09.05.05, Rafiqul Islam 70 (DUH). Cultivated.

Dalbergia volubilis Roxb., Pl. Corom. 2: 48, t. 191 (1805). Local names: Dad bari (b), Purangbuphag (t). A scandent shrub. Representative specimen: Pathachhara, 11.09.06, Rafiqul Islam 311 (DUH).

Desmodium gangeticum (L.) DC., Prodr. 2: 327 (1825). Hedysarum gangeticum L., Sp. Pl.: 746 (1753). Local name: Satpani (b). A suberect undershrub. Representative specimen: Halfchhari, 17.09.06, Rafiqul Islam 362 (DUH).

Desmodium polycarpum DC., Prodr. 2: 334 (1825). An undershrub. Representative specimen: Pathachhara, 09.05.05, Rafiqul Islam 76 (DUH).

Desmodium pulchellum (L.) Benth., Fl. Hongk.: 83 (1861). Hedysarum pulchellum L. in Roxb., Fl. Ind. 3: 361 (1832). Local name: Juta-salpani (b). A shrub. Representative specimen: Ramgarh, 11.05.05, Rafiqul Islam 63 (DUH).

Erythrina ovalifolia Roxb., Fl. Ind. 3: 251 (1832). Local name: Mandar (b). A deciduous, small tree. Representative specimen: Pathachhara, 22.12.05, Rafiqul Islam 199 (DUH).

Flemingia strobilifra R. Br. in Ait., Hort. Kew. ed. 2 (4): 350 (1812). An erect shrub. Representative specimen: Halfchhari, 25.12.05, Rafiqul Islam 200 (DUH).

Mucuna pruriens (L.) DC., Prodr. 2: 405 (1825). Dolichos pruriens L. in Stickman, Dis. Herb. Fl. Amboin.: 23 (1754). Local names: Al-kushi (b), Amukhatubupang (t). A large climber. Representative specimen: Ramgarh, 09.05.05, Rafiqul Islam 61 (DUH).

Phaseolus trilobatus Hook. f., Fl. Brit. Ind. 2: 201 (1876). Local name: Mugani (t). Twiner, usually herbaceous. Representative specimen: Pathachhara, 22.12.05, Rafiqul Islam 195 (DUH).

Pueraria phaseoloides (Roxb.) Benth., J. Linn. Soc. Bot. 9: 125 (1867). Dolichos phaseoloides Roxb., Fl. Ind. 3: 316 (1832). A herbaceous, pubescent climber. Representative specimen: Ramgarh, 21.12.05, Rafiqul Islam 70 (DUH).

Uraria lagopodiodes (L.) Desv., Mem. Soc. Linn. Paris 4: 309 (1829). Hedysarum lagopodiodes L., Sp. Pl. 1198 (1753). Local name: Latachakuley (b). A creeping, woody herb. Representative specimen: Ramgarh, 09.05.05, Rafiqul Islam 57 (DUH).

\section{Lythraceae}

Daubanga grandiflora Roxb., Fl. Ind. 2: 503 (1832). Local name: Kasshabupang (t). A large tree. Representative specimen: Halfchhari, 07.05.05, Rafiqul Islam 19 (DUH). 
Lagerstroemia parviflora Roxb., Pl. Corom. 1: 47, t. 66 (1795). Local names: Tila jalifung (t), Tila jarul (b). A small, bushy tree. Representative specimen: Pathachhara, 11.09.06, Rafiqul Islam 316 (DUH).

Lagerstroemia speciosa (L.) Pers., Syn. 2: 72 (1807). Munchausia speciosa L., Mant. Pl. 2: 243 (1771). Local name: Jarul (b). A large, deciduous tree. Representative specimen: Ramgarh, 11.05.05, Rafiqul Islam 144 (DUH).

Woodfordia fruticosa Kurz, Journ. As. Soc. Beng. 11: 56 (1871). A shrub. Representative specimen: Pathachhara, 22.12.05, Rafiqul Islam 191 (DUH).

\section{Myrtaceae}

Psidium guajava L., Sp. Pl.: 470 (1753). Local name: Piyara (b). Large shrub or small tree. Representative specimen: Pathachhara, 11.09.06, Rafiqul Islam 339 (DUH). Cultivated.

Syzygium claviflorum (Roxb.) A.M. Cowan \& J.M. Cowan, Trees N. Bengal: 67 (1929). Eugenia claviflora Roxb., Fl. Ind. 2: 488 (1832). Local name: Khorula jam (b). A tree. Representative specimen: Halfchhari, 07.05.05, Rafiqul Islam 15 (DUH).

Syzygium fruticosum (Roxb.) DC., Prodr. 3: 260 (1828). Eugenia fruticosa Roxb., Fl. Ind. 2: 87 (1832). Local name: Titi jam (b). A small tree. Representative specimen: Ramgarh, 11.05.05, Rafiqul Islam 127 (DUH).

Syzygium jambos (L.) Alston in Trimen Handb. Fl. Ceylon 6: 115 (1931). Eugenia jambos L., Sp. Pl.: 470 (1753). Local name: Gulab jam (b). A medium-sized tree. Representative specimen: Halfchhari, 07.05.05, Rafiqul Islam 30 (DUH). Cultivated.

Syzygium malaccense (L.) Merr. \& L. M. Perry, J. Arnold Arbor. 19: 215 (1938). Eugenia malaccensis L., Sp. Pl.: 470 (1753). Local name: Bon jamrul (b). A shrub or small tree. Representative specimen: Pathachhara, 10.05.05, Rafiqul Islam 103 (DUH).

Syzygium samarangense (Blume) Merr. \& L. M. Perry, J. Arnold Arbor. 19: 115, 216 (1938). Myrtus samarangensis Blume, Bijdr.: 1084 (1826). Local name: Jamrul (b). A small tree. Representative specimen: Pathachhara, 10.05.05, Rafiqul Islam 97 (DUH). Cultivated.

\section{Onagraceae}

Ludwigia adscendens (L.) Hara, J. Jap. Bot. 28: 290 (1953). Jussiaea abyssinica L., Mant. 1: 69 (1767). Local name: Keshardam (b). A creeping aquatic herb. Representative specimen: Halfchhari, 25.12.05, Rafiqul Islam 223 (DUH).

Ludwigia hyssopifolia (G. Don) Exell., Garica de Orta 5: 471 (1957). Jussiaea hyssopifolia G. Don, Gen. Syst. 2: 693 (1832). A branched herb. Representative specimen: Ramgarh, 11.05.05, Rafiqul Islam 153 (DUH). 


\section{Melastomataceae}

Melastoma malabathricum L., Sp. Pl.: 390 (1753). Local names: Datranga, Lutki (b). A shrub. Representative specimen: Ramgarh, 11.05.05, Rafiqul Islam 128 (DUH).

\section{Combretaceae}

Anogeissus acuminata (Roxb. ex DC.) Guill. \& Perr., Fl. Seneg. Tent. 1: 280 (1832). Conocarpus acuminatus Roxb. ex DC., Prodr. 3: 16 (1828). Local names: Chakua (b), Hiuri (t). A tree. Representative specimen: Pathachhara, 10.05.05, Rafiqul Islam 103 (DUH); Ramgarh, 21.12.05, Rafiqul Islam 173 (DUH).

Calycopteris floribunda (Roxb.) Lamk., Enc. Meth. Bot. Suppl. 2: 41 (1811). Getonia floribunda Roxb., Pl. Corom. 1: 61, t. 87 (1798). Local name: Goache lata (b). A dense shrub. Representative specimen: Halfchhari, 07.05.05, Rafiqul Islam 14 (DUH).

Combretum acuminatum Roxb., Fl. Ind. ed. 2: 228 (1824). A large, scandent shrub. Representative specimens: Pathachhara, 22.12.05, Rafiqul Islam 190 (DUH); Ramgarh, 11.05.05, Rafiqul Islam 163 (DUH).

Terminalia arjuna (Roxb. ex DC.) Wt. \& Arn., Prodr.: 314 (1834). Pentaptera arjuna Roxb. ex DC., Prodr. 3: 14 (1828). Local name: Arjun (b). A medium-sized tree. Representative specimen: Ramgarh, 11.05.05, Rafiqul Islam 165 (DUH). Cultivated.

Terminalia bellirica (Gaertn.) Roxb., Pl. Corom. 2: 54, t. 198 (1805). Myrobalanus bellerica Gaertn., De. Fruct. Semi. 2: 90, t. 97 (1791). Local names: Bohera (b), Shiba (t). A tree. Representative specimen: Halfchhari, 07.05.05, Rafiqul Islam 05 (DUH).

Terminalia chebula Retz., Obs. Bot. 5: 31 (1789). Local name: Haritaki (b). A large tree. Representative specimen: Pathachhara, 10.05.05, Rafiqul Islam 99 (DUH).

\section{Sapindaceae}

Cardiospermum halicacabum L., Sp. Pl. ed. 1: 366 (1753). Climbing herb. Representative specimen: Ramgarh, 11.05.05, Rafiqul Islam 150 (DUH).

\section{Euphorbiaceae}

Acalypha indica L., Sp. Pl.: 1003 (1753). Local name: Muktajhuri (b). A small robust or woody herb. Representative specimen: Ramgarh, 09.09.06, Rafiqul Islam 242 (DUH).

Aporosa wallichii Hook. f., Fl. Brit. Ind. 5: 350 (1885). Local name: Kamba (t). A medium-sized tree. Representative specimen: Halfchhari, 07.05.05, Rafiqul Islam 12 (DUH).

Baccaurea ramiflora Lour., Fl. Cochinch.: 661 (1790). Local name: Latka (b). A small tree. Representative specimen: Ramgarh, 09.05.05, Rafiqul Islam 58 (DUH). 
Breynia retusa (L.) Spreng., Syst. Veg. 3: 48 (1829). Clutia retusa L., Sp. Pl.: 1024 (1753). Local name: Silpati (b). A medium-sized tree. Representative specimen: Ramgarh, 11.05.05, Rafiqul Islam 149 (DUH).

Bridelia stipularis (L.) Blume, Bijdr.: 597 (1826). Clutia stipularis L., Mant. Pl.: 127 (1767). Local names: Harinhara (b), Kantakui (t). A subscandent shrub. Representative specimen: Ramgarh, 25.12.05, Rafiqul Islam 73 (DUH).

Croton bonplandianus Bill., Adansonia 4: 339 (1864). Local name: Moricha (b). An annual herb. Representative specimens: Halfchhari, 22.12.05, Rafiqul Islam 190 (DUH); Pathachhara, 09.05.05, Rafiqul Islam 81 (DUH).

Euphorbia hirta L., Sp. Pl.: 454 (1753). Local name: Dudhia (b). An annual, usually robust, erect or ascending herb. Representative specimens: Pathachhara, 11.09.06, Rafiqul Islam 312 (DUH) ; Ramgarh, 11.05.05, Rafiqul Islam 157 (DUH).

Glochidion multiloculare (Roxb. ex Willd.) Muell.-Arg., Linnaea 32: 59 (1863). Agyneia multilocularis Roxb. ex Willd., Neue Schr. Ges. Naturf. Freunde Berlin 4: 206 (1803). Local name: Keura (t). A small tree. Representative specimen: Ramgarh, 09.05.05, Rafiqul Islam 44 (DUH).

Macaranga denticulata (Blume) Muell.-Arg. in DC., Prodr. 15 (2): 1000 (1886). Mappa denticulata Blume, Bijdr.: 625 (1825). Local name: Bura (t). A small, ever-green tree. Representative specimen: Ramgarh, 09.05.05, Rafiqul Islam 54 (DUH).

Mallotus philippensis (Lamk.) Muell.-Arg., Linnaea 34 (1): 196 (1865). Croton philippensis Lamk., Encycl. Meth. 5: 298 (1804). Local name: Moinbura (b). A mediumsized tree. Representative specimen: Pathachhara, 10.05.05, Rafiqul Islam 96 (DUH).

Phyllanthus embelica L., Sp. Pl.: 982 (1753). Local name: Amloki (b). A small or middle-sized tree. Representative specimen: Pathachhara, 10.05.05, Rafiqul Islam 113 (DUH). Cultivated.

Phyllanthus fraterous Webster, Contr. Gray Herb. 176: 53 (1955). Phyllanthus niruri senu Hook. f., Fl. Brit. Ind. 5: 298 (1887). Local name: Bhui amla (b). A herb. Representative specimen: Pathachhara, 22.12.05, Rafiqul Islam 186 (DUH).

Phyllanthus reticulatus Poir. in Lamk., Encycl. Meth. B. 5: 298 (1804). Local name: Sitki panku (b). A large, scandent shrub. Representative specimens: Pathachhara, 22.12.05, Rafiqul Islam 197 (DUH); Ramgarh, 21.12.05, Rafiqul Islam 181 (DUH).

Phyllanthus urinaria L., Sp. Pl.: 982 (1753). An erect, glabrous, annual herb. Representative specimen: Halfchhari, 07.05.05, Rafiqul Islam 01 (DUH).

Ricinus communis L., Sp. Pl.: 1007 (1753). Local name: Rerhi (b). An ever-green shrub. Representative specimen: Halfchhari, 07.05.05, Rafiqul Islam 21 (DUH). 


\section{Rhamnaceae}

Zizyphus mauritiana Lamk., Encycl. 3: 319 (1789). Local name: Boroi (b). A small, much branched tree. Representative specimens: Halfchhari, 07.05.05, Rafiqul Islam 13 (DUH); Pathachhara, 22.12.05, Rafiqul Islam 198 (DUH).

\section{Leeaceae}

Leea aequata L., Mant. Pl. 1: 124 (1767). Leea hirta Roxb., Fl. Ind. 2: 469 (1824). Local name: Pagolgota gach (b). A shrub. Representative specimen: Ramgarh, 09.09.06, Rafiqul Islam 250 (DUH).

\section{Vitaceae}

Cayratia trifolia (L.) Domin, Biblioth. Bot. 89: 371 (1927). Vitis trifolia L., Sp. Pl.: 203 (1753). Local name: Amal-lata (b). A slender, herbaceous climber with swollen rootstock. Representative specimen: Halfchhari, 17.09.06, Rafiqul Islam 393 (DUH).

Cissus quadrangularis L., Syst. Nat. ed. 12 (2): 124 (1767). Local names: Harjora lata, Kumor lata (b). A herbaceous plant. Representative specimen: Ramgarh, 11.05.05, Rafiqul Islam 124 (DUH).

Cissus repens Lamk., Encycl. Math. Bot. 1: 31 (1783). Local names: Jangli angur (b), Marmaria-pata (t). A large, glabrous, herbaceous climber with quadrangular stem. Representative specimens: Pathachhara, 10.05.05, Rafiqul Islam 104 (DUH); Ramgarh, 21.12.05, Rafiqul Islam 169 (DUH).

\section{Burseraceae}

Bursera serrata Wall. ex Colebr., Trans. Linn. Soc. 15: 361, t. 4 (1827). Local name: Nule (b). A medium-sized tree. Representative specimens: Halfchhari, 07.05.05, Rafiqul Islam 03 (DUH); Pathachhara, 11.09.06, Rafiqul Islam 341 (DUH).

\section{Anacardiaceae}

Lannea coromandelica (Houtt.) Merr., J. Arnold Arbor. 19: 353 (1938). Dialium coromandelicum Houtt., Nat. Hist. 2: 39, t. 5, f. 2 (1774). Local names: Jika (t), Kaphila (m). A medium-sized, deciduous tree. Representative specimens: Pathachhara, 09.05.05, Rafiqul Islam 85 (DUH); Ramgarh, 11.05.05, Rafiqul Islam 156 (DUH).

Spondias pinnata (L. f.) Kurz, Pegu Rep. A. 44 (1875). Local name: Bonamra (b). A medium-sized to tall tree. Representative specimen: Halfchhari, 25.12.05, Rafiqul Islam 207 (DUH). 


\section{Meliaceae}

Aphanamixis polystachya (Wall.) Parker, Ind. For. 57: 486 (1931). Aglaia polystachya Wall. in Roxb., Fl. Ind. 2: 429 (1824). Local name: Royna (b). A tree with dense spreading crown. Representative specimens: Halfchhari, 25.12.05, Rafiqul Islam 225 (DUH); Pathachhara, 09.05.05, Rafiqul Islam 80 (DUH).

Melia azedarach L., Sp. Pl.: 384 (1753). Local name: Gora nim (b). A medium-sized tree. Representative specimen: Ramgarh, 09.05.05, Rafiqul Islam 62 (DUH). Cultivated.

\section{Rutaceae}

Aegle marmelos (L.) Corr., Trans. Linn. Soc. 5: 222 (1800). Crateva marmelos L., Sp. Pl.: 444 (1753). Local name: Bel (b). A small, deciduous tree. Representative specimen: Pathachhara, 10.05.05, Rafiqul Islam 110 (DUH). Cultivated.

Glycosmis pentaphylla (Retz.) A. DC., Prodr. 1: 538 (1824). Limonia pentaphylla Retz., Obs. Bot. 5: 24 (1788). Local name: Datmajon (b). A shrub or small tree. Representative specimens: Halfchhari, 25.12.05, Rafiqul Islam 226 (DUH); Ramgarh, 11.05.05, Rafiqul Islam 141 (DUH).

Micromelum minutum (Forst. f.) Wight \& Arn., Prodr. 1: 448 (1834). Limonia minutum Forst. f., Prodr.: 33 (1786). Local name: Duha (b). A bushy shrub. Representative specimen: Ramgarh, 11.05.05, Rafiqul Islam 137 (DUH).

Zanthoxylum rhetsa (Roxb.) DC., Prodr. 1: 728 (1825). Fagara rhetsa Roxb. (1820). Local name: Bajna (b). An ever-green, small tree. Representative specimen: Ramgarh, 09.05.05, Rafiqul Islam 45 (DUH).

\section{Oxalidaceae (Averrhoaceae)}

Averrhoa carambola L., Sp. Pl.: 428 (1753). Local name: Kamranga (b). A bushy tree. Representative specimens: Halfchhari, 07.05.05, Rafiqul Islam 24 (DUH); Pathachhara, 09.05.05, Rafiqul Islam 74 (DUH). Cultivated.

Oxalis corniculata L., Sp. Pl.: 435 (1753). Local name: Ambuli (b). An annual herb. Representative specimen: Halfchhari, 25.12.05, Rafiqul Islam 209 (DUH).

Oxalis corymbosa DC., Prodr. 1: 696 (1824). A stemless herb. Representative specimens: Pathachhara, 10.05.05, Rafiqul Islam 102 (DUH); Ramgarh, 21.12.05, Rafiqul Islam 178 (DUH).

\section{Apiaceae (Umbelliferae)}

Centella asiatica (L.) Urban in Mart., Fl. Bras. 11: 287 (1879). Hydrocotyle asiatica L., Sp. Pl. 1: 234 (1753). Local names: Adamoni, Thankuni (b). A perennial herb. Representative specimen: Pathachhara, 11.09.06, Rafiqul Islam 346 (DUH). 


\section{Apocynaceae}

Alstonia scholaris (L.) R. Br., Mem. Wern. Nat. Hist. S. 1: 75 (1811). Echites scholaris L., Mant. Pl. 1: 53 (1767). Local names: Chatim (b), Shidam (t). A medium-sized tree. Representative specimen: Ramgarh, 09.05.05, Rafiqul Islam 56 (DUH).

Holarrhena antidysenterica (L.) Wall. ex Decne., Prodr. 8: 413 (1844). Nerium antidysentericum L., Sp. Pl.: 54 (1753). Local name: Kurchi (b). A shrub. Representative specimen: Ramgarh, 09.09.06, Rafiqul Islam 236 (DUH).

Ichnocarpus frutescens (L.) R. Br. in Ait. f., Hort. Kew. ed. 2, 2: 69 (1811). Apocynum frutescens L., Sp. Pl.: 213 (1753). Local name: Sham lwui (t). A climbing shrub. Representative specimen: Halfchhari, 25.12.05, Rafiqul Islam 230 (DUH).

Rauwolfia serpentina (L.) Benth. ex Kurz, For. Fl. Brit. Burma 2: 171 (1877). Ophioxylon serpentinum L., Sp. Pl.: 1043 (1753). Local names: Churung (t), Sarpaganda (b). A woody herb. Representative specimen: Pathachhara, 11.09.06, Rafiqul Islam 330 (DUH).

Tabernaemontana recurva Roxb., Fl. Ind.: 226 (1832). A small shrub. Representative specimen: Pathachhara, 10.05.05, Rafiqul Islam 94 (DUH).

Wrightia arborea (Dennst.) Mabberly, Taxon 26: 533 (1977). Periploca arborea Dennst., Schlus. H. Malabar.: 13, 23 (1818). Local name: Dhudi (b). A small, deciduous tree. Representative specimen: Halfchhari, 25.12.05, Rafiqul Islam 231 (DUH).

\section{Solanaceae}

Datura metel L., Sp. Pl.: 179 (1753). Local name: Dutra (b). A stout herb. Representative specimen: Ramgarh, 09.09.06, Rafiqul Islam 274 (DUH).

Nicotiana plumbaginifolia Viv., Elench. Pl. Hort. Dinergo: 26. t. 5 (1802). Local name: Tamak (b). A slender, erect, annual herb. Representative specimen: Halfchhari, 25.12.05, Rafiqul Islam 210 (DUH). Cultivated.

Physalis minima L., Sp. Pl.: 183 (1753). Local name: Potka (t). An annual glabrous herb. Representative specimen: Halfchhari, 07.05.05, Rafiqul Islam 12 (DUH).

Solanum nigrum L., Sp. Pl.: 186 (1753). Local name: Tit begun (b). An annual erect shrub. Representative specimen: Ramgarh, 09.05.05, Rafiqul Islam 47 (DUH).

Solanum torvum Sw., Nov. Gen. Sp. Pl.: 47 (1788). Local name: Bot begun (b). A small shrub. Representative specimens: Halfchhari, 07.05.05, Rafiqul Islam 16 (DUH); Pathachhara, 09.05.05, Rafiqul Islam 82 (DUH).

Solanum violaceum Ortega, Hort. Matr. Dec.: 56 (1798). Local name: Phutki begun (b). A much branched undershrub. Representative specimen: Halfchhari, 17.09.06, Rafiqul Islam 382 (DUH). 


\section{Convolvulaceae}

Argyreia argentea (Roxb.) Choisy, Mem. Soc. Phys. Genev. 6: 418 (1833). Lettsomia argentea Roxb., Fl. Ind. ed. 2: 79 (1824). Local name: Dhumchuk (t). A climber. Representative specimen: Halfchhari, 25.12.05, Rafiqul Islam 233 (DUH).

Argyreia capitiformis (Poir.) Oostr. in van Steenis, Fl. Mal. Ser. 1, 6(6): 941 (1972). Convolvulus capitiformis Poir. in Lamk., Encylc. Suppl. 3: 469 (1814). A large climber. Representative specimen: Halfchhari, 07.05.05, Rafiqul Islam 17 (DUH).

Ipomoea aquatica Forssk., Fl. Aeg.-Arab.: 44 (1775). Local name: Kalmilata (b). A glabrous trailer on water. Representative specimen: Ramgarh, 11.05.05, Rafiqul Islam 158 (DUH).

Ipomoea fistulosa Mart. ex Choisy in DC., Prodr. 9: 349 (1845). Local names: Dholkalmi, Durakalma (b). A fistular shrub. Representative specimen: Pathachhara, 09.05.05, Rafiqul Islam 335 (DUH).

Ipomoea tricolor Cav., Ic. 3: 5, t. 208 (1794). A glabrous twiner. Representative specimens: Halfchhari, 17.09.06, Rafiqul Islam 389 (DUH); Pathachhara, 10.05.05, Rafiqul Islam 120 (DUH).

\section{Cuscutaceae}

Cuscuta reflexa Roxb., Pl. Corom. 2: 3, t. 104 (1798). Local name: Swarnalata (b). A fleshy parasite, forming dense yellow masses on small tree or shrub. Representative specimen: Ramgarh, 11.05.05, Rafiqul Islam 154 (DUH).

\section{Boraginaceae}

Cordia dichotoma Forst. f., Fl. Ins. Austr. Prodr. 18: 110 (1876). Local names: Bahoduri (b), Bahubara (t), Boula (b). A medium-sized, deciduous tree. Representative specimen: Halfchhari, 07.05.05, Rafiqul Islam 20 (DUH).

Heliotropium indicum L., Sp. Pl.: 130 (1753). Local name: Hatisur (b). An annual herb. Representative specimen: Halfchhari, 25.12.05, Rafiqul Islam 205 (DUH).

\section{Verbenaceae}

Callicarpa longifolia Lamk., Enc. Meth. 1: 403 (1798). Local name: Bormala (b). A shrub. Representative specimen: Pathachhara, 09.05.05, Rafiqul Islam 78 (DUH).

Clerodendrum viscosum Vent., Gard. Malm. 1: t. 25 (1803). Local name: Bhant (b). A perennial, woody herb to undershrub. Representative specimen: Halfchhari, 25.12.05, Rafiqul Islam 222 (DUH).

Lantana camara L., Sp. Pl.: 627 (1753). Local name: Guayganda (b). A shrub. Representative specimen: Pathachhara, 10.05.05, Rafiqul Islam 123 (DUH). 
Lippia javanica (Burm. f.) Spreng., Syst. 2: 752 (1825). An undershrub. Representative specimen: Pathachhara, 09.05.05, Rafiqul Islam 87 (DUH).

Tectona grandis L. f., Suppl. Pl.: 151 (1781). Local name: Shegun (b). A tree. Representative specimen: Ramgarh, 22.12.05, Rafiqul Islam 134 (DUH). Cultivated.

Vitex pubescens Vahl, Symb. 3: 85 (1794). A large tree. Representative specimen: Pathachhara, 10.05.05, Rafiqul Islam 100 (DUH).

\section{Lamiaceae (Labiatae)}

Anisomeles indica (L.) O. Kuntze, Rev. Gen.: 512 (1891). Nepeta indica L., Sp. Pl.: 596 (1753). A bushy undershrub. Representative specimen: Ramgarh, 21.12.05, Rafiqul Islam 164 (DUH).

Dysophylla crassicaulis Benth. in Wall., Pl. As. Rar. 1: 30 (1830). An annual herb. Representative specimen: Ramgarh, 11.05.05, Rafiqul Islam 162 (DUH).

Hyptis suaveolens (L.) Poit., Ann. Mus. Par. 7: 472, t. 29 (1806). Ballota suaveolens L., Syst. Nat. ed. 10: 1100 (1759). Local name: Tokma (b). An annual herb. Representative specimens: Halfchhari, 07.05.05, Rafiqul Islam 26 (DUH); Ramgarh, 11.05.05, Rafiqul Islam 151 (DUH).

Leucas aspera Spreng., Syst. 2: 743 (1825). Local name: Durung pata (b). An annual herb. Representative specimen: Pathachhara, 22.12.05, Rafiqul Islam 187 (DUH).

Ocimum tenuiflorum L., Sp. Pl.: 597 (1753). Local name: Kalo tulshi (b). A much branched, soft hairy, perennial herb. Representative specimens: Halfchhari, 07.05.05, Rafiqul Islam 23 (DUH); Pathachhara, 09.05.05, Rafiqul Islam 91 (DUH). Cultivated.

\section{Scrophulariaceae}

Scoparia dulcis L., Sp. Pl.: 166 (1753). Local name: Bondhuna (b). A herb. Representative specimen: Pathachhara, 10.05.05, Rafiqul Islam 105 (DUH).

\section{Acanthaceae}

Ecbolium linnaenum Kurz, Journ. As. Soc. Beng.: 2: 75 (1871). Local name: Uduzha (b). A shrub. Representative specimen: Halfchhari, 07.05.05, Rafiqul Islam 22 (DUH).

Hygrophila salicifolia (Vahl) Nees in Wall., Pl. As. Rar. 3: 81 (1832). Ruellia salicifolia Vahl, Symb. 3: 84 (1794). A prostrate to erect herb. Representative specimens: Halfchhari, 25.12.05, Rafiqul Islam 232 (DUH); Pathachhara, 11.09.06, Rafiqul Islam 358 (DUH).

Justicia adhatoda L., Sp. Pl.: 15 (1753). Adhatoda vasica Nees in Wall., Pl. As. Rar. 3: 103 (1832). Local name: Basak (b). A shrub. Representative specimen: Halfchhari, 25.12.05, Rafiqul Islam 229 (DUH). 
Justicia gandarussa Burm. f., Fl. Ind.: 10 (1768). Local name: Jawa ghas (b). An undershrub. Representative specimens: Halfchhari, 25.12.05, Rafiqul Islam 238 (DUH); Pathachhara, 10.05.05, Rafiqul Islam 108 (DUH).

Lepidagathis incurva Buch.-Ham. ex D. Don, Prodr. Fl. Nepal: 119 (1825). A perennial herb. Representative specimens: Pathachhara, 11.09.06, Rafiqul Islam 355 (DUH); Ramgarh, 09.05.05, Rafiqul Islam 46 (DUH).

Rungia pectinata (L.) Nees in DC., Prodr. 11: 469 (1847). Justicia pectinata L., Amoen. Acad. 4: 293 (1759). A much branched, prostrate or suberect herb. Representative specimen: Pathachhara, 09.05.05, Rafiqul Islam 91 (DUH).

Thunbergia grandiflora (Roxb. ex Rottler) Roxb., Bot. Reg. 6: t. 495 (1820). Flemingia grandiflora Roxb. ex Rottler, Ges. Naturf. Freund Berlin Neue Schriften 4: 202 (1803). Local name: Nil lata (b). A climber. Representative specimens: Pathachhara, 11.09.06, Rafiqul Islam 357 (DUH); Ramgarh, 11.05.05, Rafiqul Islam 145 (DUH).

\section{Bignoniaceae}

Oroxylum indicum (L.) Kurz, For. Fl. Brit. Burma 2: 237 (1877). Bignonia indica L., Sp. Pl.: 625 (1753). Local names: Kanaidengi, Konak, Sona (b). A medium-sized tree. Representative specimen: Ramgarh, 09.05.05, Rafiqul Islam 67 (DUH).

Stereospermum colais (Buch.-Ham. ex Dillw.) Mabberley, Taxon 27: 553 (1979). Bignonia colais Buch.-Ham. ex Dillw. (1839). Local name: Dharmar (b). A large, deciduous tree. Representative specimen: Ramgarh, 11.05.05, Rafiqul Islam 125 (DUH).

\section{Rubiaceae}

Boreria articularis (L. f.) Will., Bull. Herb. Bios. Ser. 2, 5: 956 (1905). Spermacoce articularis L. f., Suppl.: 119 (1781). Local name: Thitulon (t). An annual herb. Representative specimen: Halfchhari, 07.05.05, Rafiqul Islam 11 (DUH).

Hedyotis scandens Roxb., Fl. Ind. 1: 369 (1820). A climbing shrub. Representative specimen: Halfchhari, 17.09.06, Rafiqul Islam 371 (DUH).

Ixora acuminata Roxb., Fl. Ind. 1: 383 (1820). An undershrub. Representative specimen: Ramgarh, 09.05.05, Rafiqul Islam 55 (DUH).

Ixora cuneifolia Roxb., Fl. Ind. 1: 380 (1820). An evergreen shrub. Representative specimen: Halfchhari, 17.09.06, Rafiqul Islam 367 (DUH).

Paederia foetida L., Mant. Pl. 1: 52 (1767). Local name: Gandha badhuli (b). A slender climber. Representative specimen: Ramgarh, 09.05.05, Rafiqul Islam 72 (DUH).

Psychotria adenophylla Wall. in Roxb., Fl. Ind. 2: 166 (1824). Local name: Moshakbupang (t). A low shrub. Representative specimen: Halfchhari, 07.05.05, Rafiqul Islam 18 (DUH). 
Randia dumetorum (Retz.) Poir. in Lamk., Encycl. Suppl. 2: 824 (1812). Garaenia dumetorum Retz., Obs. Bot. 2: 14 (1781). Local name: Manakata (b). A shrub. Representative specimen: Halfchhari, 17.09.06, Rafiqul Islam 366 (DUH).

Stephegyne diversifolia Hook. f., Fl. Brit. Ind. 3: 26 (1873). Local name: Khom ghas (b). A small tree. Representative specimen: Halfchhari, 25.12.05, Rafiqul Islam 227 (DUH).

\section{Asteraceae (Compositae)}

Ageratum conyzoides L., Sp. Pl.: 839 (1753). Local names: Dulkuri, Hialmuti (b). An annual herb. Representative specimen: Ramgarh, 09.05.05, Rafiqul Islam 51 (DUH).

Blumea lacera (Burm. f.) DC. in Wight, Contrib. Bot. Ind.: 14 (1834). Conyza lacera Burm. f., Fl. Ind.: 180, t. 59 (1768). Local name: Kuksung (b). An erect, annual, aromatic herb. Representative specimen: Pathachhara, 10.05.05, Rafiqul Islam 93 (DUH).

Elephantopus scaber L., Sp. Pl.: 814 (1753). Local name: Mulasus (b). A perennial herb. Representative specimen: Halfchhari, 25.12.05, Rafiqul Islam 235 (DUH).

Enhydra fluctuans Lour., Fl. Cochinch.: 511 (1790). Local name: Helencha (b). A profusely branched, annual aquatic herb. Representative specimen: Halfchhari, 25.12.05, Rafiqul Islam 213 (DUH).

Grangea maderaspatana (L.) Poir., Enc. Suppl. 2: 825 (1811). Artemisia maderaspatana L., Sp. Pl.: 849 (1753). An annual herb. Representative specimen: Ramgarh, 11.05.05, Rafiqul Islam 161 (DUH).

Mikania cordata (Burm. f.) B.L. Robinson, Contrib. Gray Herb. 104: 65 (1934). Eupatorium cordatum Burm. f., Fl. Ind.: 176 (1768). Local name: Asamlata (b). A perennial herb. Representative specimen: Ramgarh, 21.12.05, Rafiqul Islam 171 (DUH).

Sonchus oleraceus Hook. f., Fl. Brit. Ind. 3: 414 (1882). An annual, milky herb. Representative specimen: Pathachhara, 22.12.05, Rafiqul Islam 186 (DUH).

Spilanthes calva DC. in Wight, Contrib. Bot. Ind.: 19 (1834) Spilanthes acmella auct. non Linn. Merr. (1774). Local name: Hampui (t). An annual herb. Representative specimen: Halfchhari, 07.05.05, Rafiqul Islam 28 (DUH).

\section{Liliopsida (Monocots)}

\section{Arecaceae (Palmae)}

Caryota urens L., Sp. Pl.: 1189 (1753). Trunk solitary, annulate, erect, up to $12 \mathrm{~m}$ tall. Representative specimen: Ramgarh, 09.09.06, Rafiqul Islam 268 (DUH).

Didymosperma nanum H. Wendl. \& Drude in Kerchov., Palm.: 243 (1878). A low palm. Representative specimen: Halfchhari, 17.09.06, Rafiqul Islam 398 (DUH). 


\section{Araceae}

Pothos scandens L., Sp. Pl.: 963 (1753). Local name: Hatilata (b). A climbing aroid. Representative specimen: Halfchhari, 17.09.06, Rafiqul Islam 388 (DUH).

Typhonium trilobatum (L.) Schott., Wien. Zeitschr. 3: 72 (1829). Arum trilobatum L., Sp. Pl.: 934 (1753). Local name: Gondogi (t). A tuberous climber. Representative specimen: Pathachhara, 11.09.06, Rafiqul Islam 323 (DUH).

\section{Commelinaceae}

Commelina benghalensis L., Sp. Pl.: 41 (1753). Local name: Dholpata (b). A slender herb. Representative specimen: Halfchhari, 25.12.05, Rafiqul Islam 209 (DUH).

Commelina diffusa Burm. f., Fl. Ind.: 18, t. 7, 2 (1768). An annual, slender herb. Representative specimens: Halfchhari, 17.09.06, Rafiqul Islam 391 (DUH); Pathachhara, 09.05.05, Rafiqul Islam 92 (DUH).

Murdania nudiflora (L.) Brenan, Kew Bull. 7: 189 (1952). Commelina nudiflora L., Sp. Pl.: 41 (1753). Local name: Kanduli (t). An annual, diffuse herb. Representative specimen: Ramgarh, 11.05.05, Rafiqul Islam 159 (DUH).

\section{Cyperaceae}

Cyperus compressus L., Sp. Pl. ed. 1: 46 (1753). Local name: Chancha (t). An annual herb, tufted root. Representative specimens: Halfchhari 25.12.05, Rafiqul Islam 218 (DUH); Ramgarh, 11.05.05, Rafiqul Islam 143 (DUH).

Cyperus diffusus Vahl, Enum. Pl. 2: 321 (1806). A perennial herb. Representative specimens: Halfchhari, 07.05.05, Rafiqul Islam 10 (DUH); Pathachhara, 10.05.05, Rafiqul Islam 159 (DUH).

Cyperus distans L. f., Suppl. Pl.: 103 (1781). Local name: Panimalancho (b). A perennial herb with short knotty rhizome. Representative specimen: Pathachhara, 22.12.05, Rafiqul Islam 182 (DUH).

Cyperus iria L., Sp. Pl. ed. 1: 45 (1753). An annual or rarely perennial herb. Representative specimen: Halfchhari, 17.09.06, Rafiqul Islam 395 (DUH).

Cyperus michelianus (L.) Link., Hort. Bot. Berol. Descr. 1: 303 (1827). Scirpus michelianus L., Sp. Pl.: 45 (1753). An annual herb. Representative specimen: Pathachhara, 22.12.05, Rafiqul Islam 184 (DUH).

Cyperus rotundus L., Sp. Pl.: 45 (1753). Local name: Motha ghas (b). Perennial grass. Representative specimen: Ramgarh, 21.12.05, Rafiqul Islam 174 (DUH).

Diplacrum caricinum R. Br., Prodr. Fl. Nov. Holl.: 241 (1810). An annual, small and slender herb. Representative specimen: Pathachhara, 11.09.06, Rafiqul Islam 305 (DUH). 
Fimbristylis acuminata Vahl, Enum. Pl. 2: 285 (1806). An annual herb. Representative specimen: Pathachhara, 11.09.06, Rafiqul Islam 207 (DUH).

Fimbristylis cymosa R. Br., Prodr. Fl. Nov. Holl.: 228 (1810). Rhizomatous, perennial herb. Representative specimen: Ramgarh, 09.05.05, Rafiqul Islam 52 (DUH).

Themda quadrivalvis (L.) O. Kuntze, Rev. Gen. Pl. 2: 793 (1891). Andropogon quadrivalvis L. in Merr., Syst. Veg. ed. 13: 758 (1774). An annual herb. Representative specimen: Ramgarh, 09.05.05, Rafiqul Islam 60 (DUH).

\section{Poaceae (Gramineae)}

Arundo donax L., Sp. Pl. ed. 1: 81 (1753). Local name: Gabanal (t). A perennial, tall and stout grass. Representative specimen: Halfchhari, 17.09.06, Rafiqul Islam 395 (DUH).

Axonopus compressus (Sw.) P. Beauv., Ess. Agrost. 12 (154): 167 (1812). Milium compressum Sw., Prod.: 24 (1788). A perennial, tufted herb. Representative specimen: Halfchhari, 17.09.06, Rafiqul Islam 375 (DUH).

Chrysopogon aciculatus (Retz.) Trin., Fund. Agrost.: 188 (1820). Andropogon aciculatus Retz., Obs. Bot. 5: 22 (1989). A glabrous herb. Representative specimen: Ramgarh, 21.12.05, Rafiqul Islam 175 (DUH).

Cynodon dactylon (L.) Pers., Syn. Pl. ed. 1: 85 (1805). Panicum dactylon L., Sp. Pl.: 58 (1753). Local name: Durba (b). A creeping herb. Representative specimen: Ramgarh, 21.12.05, Rafiqul Islam 179 (DUH).

Cyrtococcum accrescens (Trin.) Stapf in Hook., Ic. Pl.: sub t. 3096 (1922). Panicum accrescens Trin., Sp. Gram. 1, t. 88 (1828). An annual, scrambling grass. Representative specimen: Halfchhari, 17.09.06, Rafiqul Islam 372 (DUH).

Dactyloctenium aegyptium (L.) P. Beauv., Ess. Agrost. Expl. Pl.: 15 (1812). Cynosurus aegyptius L., Sp. Pl. ed. 1, 1: 72 (1753). Local name: Makra (t). Stoloniferous, annual or short-lived perennial herb. Representative specimen: Halfchhari, 17.09.06, Rafiqul Islam 376 (DUH).

Echinochloa crus-galli (L.) P. Beauv., Ess. Agrost. 53: 161 (1812). Panicum crus-galli L., Sp. Pl. ed. 1, 1: 56 (1753). Local name: Barashyamaghas (b). An annual or perennial herb. Representative specimen: Halfchhari, 25.12.05, Rafiqul Islam 216 (DUH).

Echinochloa stagnina (Retz.) P. Beauv., Ess. Agrost.: 53, 161, 171 (1812). Panicum stagninum Retz., Osb. Bot. 5: 17 (1789). An aquatic, perennial grass. Representative specimens: Halfchhari, 17.09.06, Rafiqul Islam 388 (DUH); Pathachhara, 11.09.06, Rafiqul Islam 308 (DUH).

Eleusine indica (L.) Gaertn., De Fruct. 1: 8 (1789). Cynusurus indicus L., Sp. Pl. ed. 1: 72 (1753). Local name: Malanga kuri (b). An annual, tufted herb. Representative specimen: Halfchhari, 17.09.06, Rafiqul Islam 371 (DUH). 
Imperata cylindrica (L.) Reaeschel, Nom. Bot. ed. 3: 10 (1797). Lagurus cylindricus L., Syst. Nat. ed. 10: 878 (1759). Local names: Son, Ulukhar (b). A perennial, rhizomatous grass. Representative specimen: Ramgarh, 11.05.05, Rafiqul Islam 146 (DUH).

Melocanna baccifera (Roxb.) Kurz, Prelim. Rep. For. Veg. Pegu App. B. 94 (1875). Bambusa baccifera Roxb., Pl. Corom. 3: 38, 243 (1819). Local names: Muli (b), Nail (t), Paiyya (m), Tarai (t). Diffusely clumped, sympodial bamboo. Representative specimens: Pathachhara, 22.12.05, Rafiqul Islam 185 (DUH); Ramgarh, 11.05.05, Rafiqul Islam 132 (DUH).

Oplismenus compositus (L.) P. Beauv., Ess. Agrost. 54: 168 (1812). Panicum compositum L., Sp. Pl. ed. 1: 57 (1753). A perennial grass. Representative specimen: Ramgarh, 21.12.05, Rafiqul Islam 176 (DUH).

Panicum montanum Roxb., Fl. Ind. 1: 315 (1820). A perennial, tufted grass. Representative specimen: Pathachhara, 11.09.06, Rafiqul Islam 313 (DUH).

Panicum repens L., Sp. Pl. ed. 2: 87 (1753). A perennial, rhizomatous grass. Representative specimen: Halfchhari, 25.12.05, Rafiqul Islam 215 (DUH).

Paspalum scrobiculatum L., Mant. 1: 29 (1767). Local name: Goicha (b). An annual herb. Representative specimen: Ramgarh, 21.12.05, Rafiqul Islam 177 (DUH).

Saccharum spontaneum L., Mant. Pl. 2: 183 (1771). Local name: Kash (b). A perennial, tall herb. Representative specimen: Halfchhari, 17.09.06, Rafiqul Islam 374 (DUH).

Setaria palmifolia (Koen.) Stapf, J. Linn. Soc. Bot. 42: 186 (1914). Panicum palmaefolium Koen. (1788). Local name: Urodhan (b). A perennial grass. Representative specimens: Pathachhara, 11.09.06, Rafiqul Islam 318 (DUH); Ramgarh, 09.09.06, Rafiqul Islam 43 (DUH).

\section{Typhaceae}

Typha elephantina Roxb., Fl. Ind. ed. 3: 506 (1832). Local name: Hogla (b). A robust herb. Representative specimen: Pathachhara, 22.12.05, Rafiqul Islam 183 (DUH).

\section{Zingiberaceae}

Alpinia nigra (Gaertn.) Burtt., Notes Roy. Bot. Gard. Edinb. 35: 213 (1977). Zingiber nigrum Gaertn. (1788). Local name: Tara (b). Stem leafy, leaves sessile or sub-sessile. Representative specimen: Halfchhari, 07.05.05, Rafiqul Islam 10 (DUH).

\section{Costaceae}

Costus speciosus (Koen.) Smith, Trans. Linn. Soc. London 1: 249 (1791). Banksea speciosa Koen. in Retz., Obs. Bot. 3: 75 (1783). Local names: Gardong (t), Jongliphul 
(b). A rhizomatous herb. Representative specimen: Pathachhara, 10.05.05, Rafiqul Islam 106 (DUH).

\section{Marantaceae}

Schumannianthus dichotoma (Roxb.) Gagnep., Bull. Soc. Bot. Fr. 51: 176 (1904). Clinogyne dichotoma (Roxb.) Salisb. ex Benth. in Benth. \& Hook. f., Gen. Pl. 3 (2): 651 (1883). Phrynium dichotomum Roxb., Asiat. Rar. 11: 324 (1810). Local names: Mukta, Mustak (b). A tall, monoecious palm. Representative specimen: Ramgarh, 09.09.06, Rafiqul Islam 298 (DUH).

\section{Pontederiaceae}

Eichhornia crassipes (Mart.) Solms in A. DC., Mon. Phan. 4: 527 (1883). Pontederia crassipes Mart., Nov. Gen. Sp.: 9, t. 4 (1823). Local name: Kachuripana (b). A freefloating herb. Representative specimen: Pathachhara, 11.09.06, Rafiqul Islam 343 (DUH).

Monochoria hastata (L.) Solms. in A. DC., Mon. Phan. 4: 523 (1883). Pontederia hastata L., Sp. Pl.: 288 (1753). An aquatic, emergent herb. Representative specimen: Ramgarh, 09.05.05, Rafiqul Islam 33 (DUH).

\section{Liliaceae}

Asparagus acerosus Roxb., Fl. Ind. 2: 150 (1832). Local name: Shatamuli (b). A perennial subscandent undershrub. Representative specimen: Pathachhara, 09.05.05, Rafiqul Islam 95 (DUH).

Curculigo orchioides Gaertn., De Fruct. 1: 63, t. 13 (1788). Local name: Langtipata (b). A slender herb with elongated rhizome. Representative specimen: Ramgarh, 09.05.05, Rafiqul Islam 40 (DUH).

Molineria recurvata (Dryand.) Herbert, Amaryl.: 84 (1834). Curculigo recurvata Dryand. (1811). Local names: Luruk (t), Satipata (b). A stout herb with tuberous rootstocks. Representative specimen: Ramgarh, 09.05.05, Rafiqul Islam 48 (DUH).

\section{Agavaceae}

Dracaena spicata Roxb., Fl. Ind. 2: 157 (1824). An erect shrub. Representative specimen: Pathachhara, 11.09.06, Rafiqul Islam 316 (DUH). Cultivated.

\section{Smilacaceae}

Smilax zeylanica L., Sp. Pl.: 1029 (1753). Local name: Kumarilata (b). A stout climber. Representative specimen: Ramgarh, 09.05.05, Rafiqul Islam 53 (DUH). 


\section{Dioscoreaceae}

Dioscorea bellophylla (Prain) J.O. Voigt ex Haines, For. Fl. Choto Nagpur: 530 (1910). Dioscorea nummularia var. belophyla Prain, Bengal Pl. 2: 802 (1903). Local name: Shora alu (b). A perennial climber. Representative specimen: Ramgarh, 11.05.05, Rafiqul Islam 131 (DUH).

Dioscorea pentaphylla L., Sp. Pl.: 1032 (1753). Local name: Jhum alu (b). A climber. Representative specimen: Pathachhara, 10.05.05, Rafiqul Islam 112 (DUH).

\section{Orchidaceae}

Brachycorythis helferi (Reichb. f.) Summerh., Kew Bull. 10: 235 (1955). Gymbadenia helferi Reichb. f., Flora 55: 276 (1872). An annual, herb. Representative specimens: Halfchhari, 17.09.06, Rafiqul Islam 396 (DUH); Ramgarh, 11.05.05, Rafiqul Islam 152 (DUH).

Camarotis pallida Lindl., Journ. Linn. Soc. 3: 37 (1859). A perennial epiphyte. Representative specimen: Pathachhara, 10.05.05, Rafiqul Islam 115 (DUH).

Cymbidium aloifolium (L.) Sw., Nova Acta Regiae Soc. Sci. Upsal. 6: 73 (1799). Epidendrum aloifolium L., Sp. Pl.: 953 (1753). A perennial epiphyte. Representative specimen: Halfchhari, 17.09.06, Rafiqul Islam 381 (DUH).

Vanda tessellata (Roxb.) Hook. f. ex G. Don in Loud., Hort. Brit.: 372 (1830). Epidendrum tessellatum Roxb., Pl. Corom. 1: 34, t. 42 (1795). An epiphytic herb. Representative specimen: Ramgarh, 09.05.05, Rafiqul Islam 31 (DUH).

\section{Acknowledgement}

The authors are grateful to the Director, Center for Advanced Studies and Research in Biological Sciences, University of Dhaka for the financial support to the field work.

\section{References}

Choudhury, M.R. 1975. Working Plan for Chittagong Hill Tracts North Forest Division for the period 196970 to 1988-89. Vol. II. Forest Department, Government of Bangladesh, pp. 1-9.

Cronquist, A. 1981. An Integrated System of Classification of Flowering Plants. Columbia University Press, New York, pp. 1-1262.

Heinig, R.L. 1925. List of Plants of Chittagong Collectorate and Hill Tracts. Darjeeling, India, 1-78 pp.

Hooker, J.D. 1872-1897. The Flora of British India. Vols 1-7 (Ind. Repr. 1973). Bishen Singh Mahendra Pal Singh, Dehra Dun, India.

Khan, M.S. and Banu, F. 1969. A taxonomic report on the angiospermic flora of Chittagong Hill Tracts-1 (Monocotyledons). J. As. Soc. Pak. 14(2): 217-224.

Khan, M.S. and Banu, F. 1972. A taxonomic report on the angiospermic flora of Chittagong Hill Tracts-2 (Dicotyledons). J. As. Soc. Bangladesh 17(2): 59-88. 
Lavlu, M.N.U. 2003. Ramgarh upazila. In: Islam, S. (ed.), Banglapedia. Vol. 7. Asiatic Society of Bangladesh, Dhaka, pp. 351-352.

Prain, D. 1903. Bengal Plants. Vols 1 \& 2. Indian Reprint 1981. Bishen Singh Mahendra Pal Singh, Dehra Dun, India.

Rizvi, S.N.H. 1969. East Pakistan District Gazetteers for Chittagong. Government of East Pakistan Survices and General Administration Department, Dhaka.

Uddin, M.Z. and Hassan, M.A. 2004. Flora of Rema-Kalenga Wildlife Sanctuary. IUCN, Bangladesh Country Office, Dhaka, Bangladesh, pp. 1-120.

Uddin, S.N., Khan, M.S., Hassan, M.A. and Alam, M.K. 1998. An annotated checklist of angiospermic flora of Sita Pahar at Kaptai in Bangladesh. Bangladesh J. Plant Taxon. 5(1): 13-46.

(Manuscript received on 13 November 2008; revised on 16 April 2009) 